\title{
Chapter 18 \\ ETUDE SUR MODELE DU TRANSPORT LITTORAL CONDITIONS DE SIMILITUDE
}

\author{
J. Valembois \\ Chef de la Division Recherches \\ Laboratoire National d'Hydraulique - Chatou
}

France

\section{INTRODUCTION}

$L^{2}$ objet de la présente note est $d^{2} 6$ tudier les conditions de similitude que $I^{\prime}$ on obtient, pour la représentation sur modèle du transport littoral, à partir de divers critères. Les résultats concernent les plages formées de sable fin, à l'exclusion des plages de galets et des zones où la vase existe en proportions importantes.

Le premier critère examiné est la reproduction du début $d^{2}$ entratnement des matériaux de fond sous l'action du mourement oscillatoire de l'eau da à la houle, la oouche limite étant supposée laminaire dans la nature et sur le modèle.

Les résultats obtenus sont ensuite étendus à la représentation du début $d^{8}$ entrainement sous l'action du courant de transport de masse perpendiculaire à la plage, même lorsque cet écoulement est turbulent au roisinage du fond.

La représentation correcte du début $d^{\mathbf{t} e n t r a f n e m e n t ~ c o n d u i t ~ p r o b a b l e m e n t ~}$ à une bonne concordance entre le modèle et la nature pour la zone située bien au large du déferlement, mais il ntest pas du tout évident (et il serait même étonnant) qu'elle permette d'obtenir sur le modele un transport littoral ressemblant au transport naturel dans les zones voisines du déferlement et près du rivage. Fn effet, les phénomènes hydrauliques sont tout à fait différents dans ces régions, où interviennent de façon prépondérante la turbulence créée par le déferlement, les courants de masse perpendiculaires et parallèles à la côte, et probablement la percolation dans la zone de l'estran, ainsi que

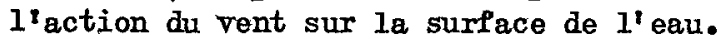

Pour aller un peu plus loin, on a essayé de définir les conditions dans lesquelles le mouvement des matériaux en suspension est correctement représenté sur le modèle.

La connaissance incomplète que $1^{*}$ on a actuellement des lois du transport littoral ne permet pas de décider a priori si les critdres retenus sont valables; cependant, on est arrivé dans divers Laboratoires, avec des modèles dont les échelles étaient voisines de celles auxquelles on aboutirait de cette façon, à représenter des transports littoraux assez semblables à ceux de la nature. On verra $d^{x}$ ailleurs que les conditions auxquelles on arrive laissent quelque liberté dans le choix des échelles. Cette liberté a probablement été utilisée dans le bon sens sur les modèles en question. 


\section{COASTAL ENGINEERING}

\section{STMLITUDE DU DEBUT D'ENTRATNEMAENT. CAS DE LA COUCHE LIMITE IAAMTAIRE.}

Si 1 on connaissait les lois du début d'entrafnement dans la nature et sur modèle, on pourrait en déduire les conditions de similitude.

Comme on ne connaft pas les lois naturelles, il faut bien partir des lois établies sur modèle*, en admettant leur extrapolation à la nature.

RHEATION DE SIMTLITUDE DE GODDET.

Goddet $[5]$ trouve, pour le début dientratnement en modèle, la loi :

$$
V_{\max }=27(\varepsilon \rho)^{2 / 3} D^{1 / 4} T^{3 / 8}
$$

(unités c.g.s.)

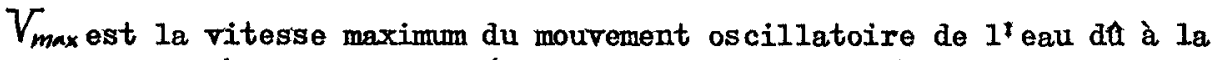
houle près du fond, immédiatement au-dessus de la couche limite,

$p$ est la masse spécifique de $1^{\text {teau, }}$

$P_{3}$ est la masse spécifique du sédiment,

$\stackrel{\mathcal{L}}{\varepsilon}=\left(\beta_{\beta}-\rho\right) / \rho$ est la densité apparente du sédiment,

$D$ est le diamètre des grains,

$T$ est la période de la houle.

Il applique cette relation à un modèle où Ia houle est reproduite en

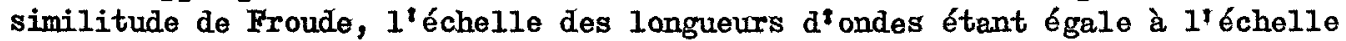
des hauteurs, dont $1^{i}$ échelle en plan peut être différente, et où $I^{*}$ échelle de

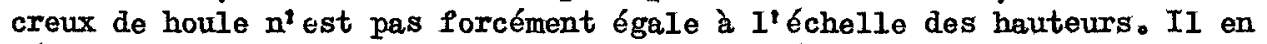
déduit une relation de similitude qui peut s'écrire sous la forme :

$$
\xi^{5 / 4}=\mu^{8 / 3} \delta D_{c}^{-4}
$$

$\xi \quad$ est $I^{*}$ échelle des profondeurs = profondeur mod/profondeur nature,

$\mu$ est $1^{\prime}$ échelle des densités apparentes des máriaux de fond,

$\delta$ est $1^{\prime}$ échelle des diamètres $D$ des matériaux de fond,

$D_{c}$ est la distorsion des creux de houle par rapport aux profondeurs = échelle des creux $/ \xi$.

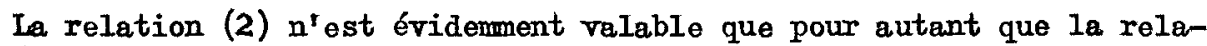
tion (1) puisse être extrapolée à la nature, ce qui est vraisemblable tant que la couche limite y reste laminaire.

* Larras $[1]$, Vincent $[2]$, Madhav Manohar [3], Lhermitte $[4]$, Goddet $[5]$ 


\section{ETUDE SUR MODELE DU TRANSPORT LITTORAL CONDITIONS DE SIMILITUDE}

REHLATION DE GODDET MODIFIEA.

Pour voir si la loi de début d'entrânement trouvée par Goddet a des chances d'être extrapolable, on peut essayer de la mettre sous une forme qui se rapproche de la forme adoptée par Shields [6] pour le début d'entrânement sous $I^{\prime}$ action $d^{\prime}$ un courant. Cette relation peut $s^{\prime}$ écrire :

$$
\frac{\tau_{0}}{\varepsilon \rho g D}=f\left(R_{*}\right)
$$

$\tau_{0}$ est la tension tangentielle exercée par l'eau sur le fond,

$R_{*}=u_{*} D / \nu$ est le nombre de Reynolds étoilé rapporté au grain,

$\nu$ étant la viscosité cinématique de I'eau et $u_{*}=\sqrt{\tau_{0} / \rho}$

la vitesse de frottement.

Nous introduirons ici un nombre sans dimension dont $I^{\prime}$ importance pour le transport solide semble considérable. C'est le rapport de $\tau_{0} / \varepsilon \rho g D$ à $R_{*}^{2}$. Il s'écrit :

$$
G=\frac{\varepsilon g \mathcal{D}^{3}}{\nu^{2}}
$$

Nous I'appellerons paramètre du grain. Il caractérise le comportement du grain dans le fluide.

La relation (3) de Shields peut s'écrire

$$
G=f\left(R_{*}\right) \times R_{*}^{2}
$$

ou, aussi bien,

$$
G=f\left(R_{*}\right)
$$

La figure 1 montre la relation de Shields (canal) sous la forme (5), ou plutot sous la forme $G=f\left(R_{*}^{2}\right)$.

On a porté sur le graphique les points expérimentaux de Goddet (houle). Pour cela, on a calculé la tension tangentielle maximum $\tau_{0}$ au moyen de la théorie de la couohe limite laminaire oscillatoire $[7]$.

$$
\tau_{0}=\nu \rho V_{\max } / \sqrt{\nu T}
$$

On a alors :

$$
R_{*}^{2}=\frac{V_{\max } D}{\nu} \times \frac{D}{\sqrt{\nu T^{2}}}
$$




\section{COASTAL ENGINEERING}

On voit que les points expérimentaux se groupent raisonnablement sur une droite pour $R_{*}^{2}$ compris entre 1 et $3 \times 10^{2}$. Si $1^{1}$ on admet comme équation de cette droite*

$$
G=10 R_{*}^{8 / 3}
$$

cela revient à :

$$
V_{\max }=0,175 g^{3 / 4} \varepsilon^{3 / 4} D^{1 / 4} T^{1 / 2}
$$

qui ressemble diassez près (et ce $n^{1}$ est pas étonnant) à la loi (1) quªdmet Goddet.

La loi (6) donne comme condition de similitude :

$$
\xi=\mu^{3} \delta D_{c}^{-4}
$$

Nous ne nous étonnerons pas non plus que cette relation ressemble de fort près à la relation $(2)$.

\section{CONDITIONS $D^{*}$ APPLICATION DE CES REALATIONS.}

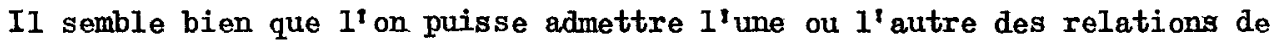
similitude, pourvu que les conditions suivantes soient remplies, aussi bien pour la nature que pour le modèle :

- Ia couche limite doit etre laminaire,

- $G=\varepsilon g D^{3} / \gamma^{2}$ doit etre inférieur à $2 \times 10^{4}$, ce qui correspond pour la nature à une dimension maximum des grains de sable de $1^{\prime}$ ordre du millimètre.

$-R_{*}^{2}=V_{\max } D^{2} / \nabla \sqrt{\nu T}$ doit otre inférieur à 400. $\left(R_{*}<20\right)$.

Cette dernière relation, pour une houle de 10 secondes, conduit à une vitesse maximum admissible de $1^{2}$ ordre de $1 \mathrm{~m} / \mathrm{s}$ pour des grains de $1 \mathrm{~mm}$, et de $4 \mathrm{~m} / \mathrm{s}$ pour des grains de $0,5 \mathrm{~mm}$.

Afin de nous affranchir de ces limites, qui sont dues simplement aux limites des conditions expérimentales des essais sur lesquels nous nous sommes basés, nous allons essayer d'utiliser la liberté importante qui reste dans le choix des éohelles. Cette liberté pourra aussi être mise à profit pour étendre le domaine d application de la similitude envisagée.

* Líquation, choisie en toute bonne foi, se trouve éliminer $\nu$ de la relation (6). C'est pourquoi on ne troure pas l'échelle des viscosités dans la relation de similitude $(7)$. 
ETUDE SUR MODELE DU TRANSPORT LITTORAL

CONDITIONS DE SIMILITUDE
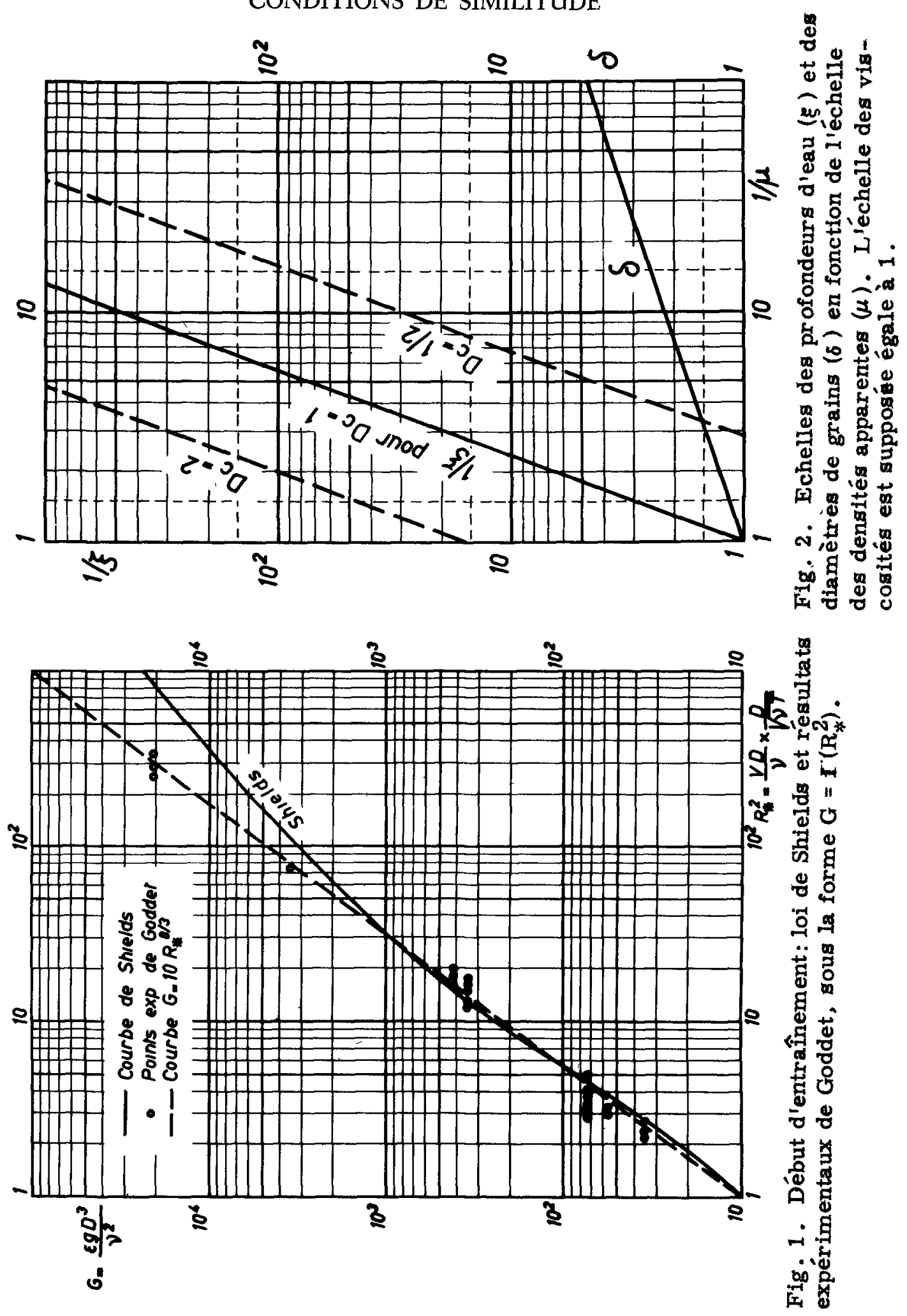


\section{COASTAL ENGINEERING}

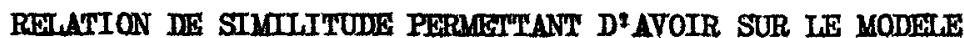 IA MAME VALEUR DE $G=\varepsilon g D^{3} / \nu^{2}$ gUE DANS IA NATURE}

Les relations (2) et (7), qui caractérisent la similitude du début $d^{2}$ entrainement dans une couche limite laminaire oscillatoire, laissent une grande liberté dans le choix des échelles, puisque nous avons une seule relation obligée entre les échelles $\xi, \mu, \delta, D_{c}, \varphi$ et $\lambda$, cette dernière

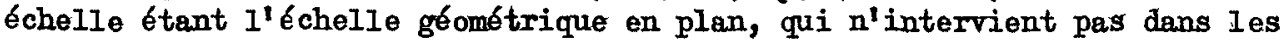
relations ci-dessus.

II nous est donc possible d*essayer de faire intervenir d'autres critères

\section{PROPRIETES DU PARMMEYRE $G$}

La conservation de la même valeur de $G=\varepsilon g D^{3} / \mathcal{\nu}^{2}$ dans le modèle que dans la nature paraft être intéressante, car ce paramètre semble avoir des propriétés remarquables.

Si, par exemple, on écrit l'Equation donnant la vitesse $W$ de chute libre $\mathrm{d}^{*} u n$ grain de forme donnée en égalant le poids apparent et la poussée, on obtient la relation :

$$
G=f\left(R_{w}\right)
$$

avec $R_{w}=W D / \mathcal{\nu}$, nombre de Reynolds correspondant à la chute libre du grair

Donc, si I'on conserve $G$, on conserve $T_{w}$, c'est-àmdire que $I^{\prime}$ on se trouve dans le même régime d'écoulement autour du grain tombant en chute libre dans $1^{*}$ eau. On retroure ici une propriété remarquable des lois de la chute d'un corps pesant dans un fluide : si deux corps de même forme (deux sphères par exemple) tombant dans le mềme fluide ont mềme poids apparent, leu $R_{w}$ de chute est le même, $c^{\prime}$ est-àmdire que leur vi tesse de chute est inversement proportionnelle à leur diamètre.

Si, d'autre part, on considère la façon dont les divers auteurs ont port en graphique les lois de $I^{2}$ entrafnement des matériaux de fond par I'eau (pour un courant unidirectionnel), on voit que le régime du matériau de fond (immobilité, début de charriage, rides, dunes, etc..) est caractérisé par la position du point représentatif du régime sur un graphique dont les coordonné sont soit $\tau_{*} / \varepsilon \rho g D$ et $R_{*}$, soit $u_{*} / w$ et $R_{*}$. Ces deux représentations s'équi valent, car on peut transformer le graphique $\left(\tau_{f} / \varepsilon \rho g D, R_{*}\right)$ en un graphique $(G$, La mềme opération peut être faite aussi bien sur le graphique $\left(u_{*} / w, R_{*}\right)$, puisque

$$
u_{*} / w=R_{*} / R_{w}=R_{*} / f(G)
$$

La correspondance entre les graphiques $\left(G, R_{*}\right)$ et $\left(u_{*} / w, R_{*}\right)$ dépendra cepen dant de la forme des grains, puisque la relation $G=f\left(R_{w}\right)$ en dépend.

Mais, pratiquement, on peut dire que le régime du matériau de fond est caractérisé par les valeurs de $G$ et de $R_{*}$. 


\section{ETUDE SUR MODELE DU TRANSPORT LITTORAL CONDITIONS DE SIMILITUDE}

Si I'on considère maintenant les diverses formules admises pour le débit transporté dans une rivière par charriage, on pourra remarquer que des formules aussi dissemblables que celles d'Einstein, de Meyer-Peter, de Frijlink (et sa forme simplifiée due à Bijker), se mettent toutes sous la forme :

$$
T G^{-1 / 2}=f\left(R_{*}^{2} G^{-1}\right)
$$

$T=q_{v} / \nu$, est un nombre que nous appellerons paramètre de transport, dans lequel $q_{p}$ est le débit en volume de matériau solide par unité de largeur du lit.

On voit que $R_{*}$ et $G$ caractérisent aussi le débit solide transporté par unité de largeur. Si ces lois sont valables, en conservant $G$ et $R_{*}$ sur un modèle, on y aurait le même débit solide en volume par unité de largeur que dans la nature*.

\section{RERLATION DE SIMIIIIUDE CONSERRANT G} suivante :

La relation de similitude correspondant à I'égalité $G_{\text {med }}=G_{\text {nat }}$ est la

$$
\delta=\mu^{-1 / 3} \varphi^{2 / 3}
$$

Rappelons que :

$\delta=\mathcal{D}_{\bmod } / D_{\text {mof est }} 1^{2} 6$ chelle des diamètres des matériaux,

$\mu$ est I"échelle des densités apparentes des matériaux,

$\varphi$ est $1^{2}$ échelle des viscosités (on pourra sourent admettre que $\varphi=1$ ). REHLATIONS DE STMULITUDE CONSERTANT $G$ ET $R *$

Les relations de similitude (7) et (10) combinées donnent alors :

$$
\begin{aligned}
& \xi=\mu^{8 / 3} D_{c}^{-4} \varphi^{2 / 3} \\
& \delta=\mu^{-1 / 3} \varphi^{2 / 3}
\end{aligned}
$$

Ces relations sont beaucoup plus restrictives que la relation (7) seule.

* Il faut noter que la relation (9) est incomplète, car les divers auteurs font intervenir aussi un coefficient tenant compte de la fraction de la force tractrice totale qui est utilisée pour le transport. Il faudrait évidemment en tenir compte dans une étude plus poussée. 


\section{COASTAL ENGINEERING}

Remarques -

a) La similitude définie par les relations (11) est plus générale qu'on ne pourrait le penser. En effet, elle résulte de la combinaison de la relation (7), établie à partir de $1^{2}$ Équation $G=10 R_{*}^{8 / 3}$, ot de Ia condition $G_{\text {mar }}=G_{\text {ma }}$

On obtiendrait les mêmes relations à partir de la condition $G_{\text {mod }}=G_{\text {nat }}$ et $d^{*}$ une relation quelconque $G=f\left(R_{*}\right)$ définissant le début d"entrainement. En effet, conserver $G$ et une relation de la forme $G=f\left(R_{*}\right)$ revient en fait à conserver $G$ et $R_{*}$ *

La similitude définie par les relations (11) consiste donc:

- à prendre le même paramètre de grain $G$ sur le modèle que dans la nature, - à prendre la même valeurr de $R_{*}$ sur le modèle que dans la nature, en admettant pour le calcul de $\tau_{0}$ que la couche limite oscillatoire est laminaire. Si la condition de début dientra inement est bien de la forme $G=f\left(R_{*}\right)$, on a

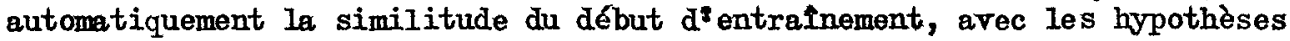
restrictives indiquées ci-dessus.

b) L'échelle des densités apparentes conditionne $1^{1}$ échelle des diamètres de grains. Si $1^{\prime}$ on prend un matériau de densité plus faible que le sable, les grains seront plus gros sur le modèle que dans la nature. (Voir fig, 2). (Nous supposons ici la viscosité peu différente dans le modèle et dans la nature).

c) L'échelle des profondeurs est assez arbitraire, puisqu'il suffit de modifier un peu l'échelle des creux de houle pour la faire varier dans de larges limites. Pour une échelle des creux de houle égale à $1^{\prime}$ échelle des profondeurs, elle dépendrait uniquement de 1'échelle des densités apparentes (fig. 2).

d) L'échelle en plan est absolument arbitraire.

On voit qu'il reste une grande liberté pour adapter le modèle à satisfaire à d'autres exigences.

\section{DEBUT D'ENTRATNEMGNT PAR LE COURANT GENERRAL DE MASSE PERPPENDICULATRE A LA PLAGE}

Longuet-Higgins [8] a calculé, pour une houle frontale, le courant de masse associé à la houle en supposant que l'écoulement reste laminaire. Ce courant porte à la côte au voisinage du fond.

Russel et Osorio [9] ont vérifié sur modèle que la formule de LonguetHiggins donne de bons résultats pour le courant au voisinage du fond, même si l'écoulement devient turbulent. Longuet-Higgins a exposé les raisons de cette concordance dans le même article. Il calcule aussi la valeur moyenne de la tension tangentielle $\tau_{0}$ sur le fond, qui, en régime purement oscillatoire est nulle, et il aboutit à une expression que nous écrirons sous la Porme :

$$
\tau_{\text {omayen }_{\text {mage }}}=\frac{\sqrt{\pi}}{2} \times \frac{\nu \rho V_{m}^{2}}{c \sqrt{\nu T}}
$$

C étant la célérité do la houlo. 


\section{ETUDE SUR MODELE DU TRANSPORT LITTORAL CONDITIONS DE SIMILITUDE}

Le $R_{*}$ correspondant est donc donné par la relation :

$$
R_{*}^{2}=\frac{\tau_{0} D^{2}}{\rho \nu^{2}}=\frac{\sqrt{\pi}}{2} \times \frac{V_{m} D}{\nu} \times \frac{D}{\sqrt{\nu T}} \times \frac{V_{m}}{C}
$$

On voit que pour conserver la valeur de ce $R_{*}$, il suffit, si $I^{*}$ on satisfait aux relations (11), de conserver sur le modèle la même valeur de $V_{m} / c$ que dans la nature, car les relations (11) sont obtenues à partir de la conservation de $V_{m} D^{2} / v \sqrt{\nu T}$. Cette condition est réalisée si $I^{\prime}$ on a une échelle des creux égale à celle des profondeurs.

On restreint ainsi beaucoup le choix des échelles. L'échelle $\delta$ des diamètres de matériaux et $I^{2}$ échelle en hauteur $\xi$ sont déterminées, dès $q u^{*}$ on a choisi $\mu$, échelle des densités apparentes (fig. 2 avec $\mathcal{D}_{c}=1$ ).

Cependant, si $1^{\prime}$ on $s^{t}$ écarte un peu de la condition de conservation de $V_{m} / C$, on change assez peu la valeur du $\mathbb{R}_{*}$ correspondant au courant de masse, mais on peut faire varier beaucoup l'échelle en hauteur, puisqu'elle varie comme $\mathcal{D}_{c}^{-4}$.

On peut remarquer que, si $I^{2}$ on partait de $I^{2}$ expression (12) pour $R_{*}$, les relations de similitude correspondant à $l a$ conservation de $G$ et $R_{*}$ seraient les relations (11), où $D_{c}^{-4}$ serait remplacé par $D_{c}^{-8}$. La distorsion des creux de houle y est encore plus importante, ce qui se comprend, puis que la tension tangentielle introduite est proportionnelle au carré de ces creux.

\section{REPRESERTATION DES TRANSPORTS EN SUSPENSION}

Il faut, dans un modèle de plage, que le profil soit correctement représenté. Des essais en canal avec le matériau choisi et les houles du modèle indiquent le profil modèle correspondant au profil naturel, et déterminent la distorsion, done l'échelle en plan à adopter.

Pour obtenir théoriquement cette distorsion, il faut trouver le phénomène déterminant dans la formation du profil. Or, le profil $\mathrm{d}^{2}$ une plage dépend de nombreux phénomènes physiques, et particulièrement de la nature plus ou moins régulière de la houle incidente (une houle irrégulière érodant 1'estran alors qu'une houle régulière le recharge), et aussi probablement [10] de la percolation près du rivage. Fnfin, et surtout semble-t-il, le profil dépend de la façon dont les matériaux mis en suspension par le déferlement sont transportés par les courants de masse. $L^{2}$ inflluence du vent est aussi très sensible.

Il est cependant probable qu'une condition nécessaire, sinon suffisante, pour $\mathrm{qu}^{\prime}$ un modèle de transport littoral soit représentatif, est que les chemins parcourus par les particules en suspension soient homologues aux parcours naturels. Pour simplifier, nous considèrerons le transport en suspension des matériaux sous $1^{*}$ action des courants de masse perpendiculaires à la plage, et nous écrirons la condition de similitude correspondante. Cette condition consiste à conserver dans le modèle la valeur naturelle du rapport suivant :

vitesse de chute / profondeur d'eau

vitesse du courant de masse / longueur perp. à la plage 


\section{COASTAL ENGINEERING.}

Nous supposerons que nous avons déjà admis la conservation de $\mathbb{R}_{*}$ et de $G$.

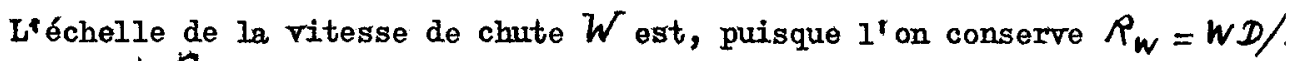
en conservant $G$,

$$
E c h(w)=\delta^{-1} \varphi
$$
Higgins,

La vitesse du courant de masse au voisinage du fond est, $d^{t}$ après Longuet-

$$
V=5 V_{m}^{2} / 4 C
$$

Son échelle est donc :

$$
\operatorname{Ech}(v)=\eta^{2} \xi^{-3 / 2}=\xi^{1 / 2} D_{c}^{2}
$$

( $\eta$ est l'échelle des crewx de houle).

Si on appelle $\lambda 1^{\prime}$ '́chelle des longueurs perpendiculairement à la plage et $\mathcal{D}_{h}=\xi / \lambda$ la distorsion géométrique correspondante, on obtient la relation de similitude suivente :

$$
D_{h}=\delta^{-1} \xi^{-1 / 2} D_{c}^{-2} \varphi
$$

Combinée avec les relations (11), cette relation devient :

$$
D_{h}=\mu^{-1}
$$

Si, au lieu de la valeur de $\tau_{0}$ correspondant à la couche limite laminaire on avait utilisé la valeur correspondant au courant de masse pour écrire la conservation de $R_{*}$, on aurait :

$$
D_{h}=\mu^{-1} D_{c}^{2}
$$

Les valeurs données par la relation (13) sont du même ordre de grandeur que les valeurs obtemues par des essais en canal. Pour un matériau de densité absolue $1,4 \mathrm{par}$ exemple, la distorsion à adopter est 4.

\section{REFFERRENCES BIBLTOCRAPHI QUUS.}

1. Iarras, J. (1956). Effets de la houle et du clapotis sur les fonds de sable - 4ème Journées de 1'Hydraulique - Tome 2 - pp. 579-589.

2. Vincent, G. F. (1958). Contribution to the study of sediment transport on a horizontal bed due to wave action - 6th Conf. on Coastal Eng. pp. 326-355.

3. Madhar Manohar, (1955). Mechanics of Bottom sediment movement du to wave action - Beach Erosion Board rech. Mem. $n^{0} 75$.

4. Lhermitte, $P_{\text {。 }}$ (1958). Contribution à l'étude de la couche limite des houles progressives. Pub1. no 136 du C.0.E.C. - Paris - Imp. Nat.

5. Goddet, J. (1959). Étude du début d'entrânement des matériaux mobiles sous l'action de la houle - La Houille Blanche n* 2 - 1960. 


\section{ETUDE SUR MODELE DU TRANSPORT LITTORAL \\ CONDITIONS DE SIMILITUDE}

6. Shields, 1. (Voir par ex. Rouse : Fngineering Hydraulics, p. 790).

7. Valembois, J. (1956). Quelques considérations sur la similitude dans les essais de houle sur modèle - 4ème Journées de l'Hydraulique - Tome 1 pp. 252-256.

8. Longuet-Higgins, M.S. (1953). Masx Transport in Water Waves - Phil. Trans。 Roy. Soc. London, Series A, n 903, vol. 245, pp. 553-581.

9. Russel, R.C.H. and Osorio, J.D.C. (1958). An experimental investigation of drift profiles in a closed channel - 6th Conf. On Coastal Eng. pp. 171-193.

10. Silvester, R. (1959). Ingineering aspects of Coastal Sediment Movement Proc. A.S.C.E. Vol. 85 - WW3 - pp. 11-40. 